\title{
PRACTICAL EVALUATION OF A REFERENCE ARCHITEC- TURE FOR THE MANAGEMENT OF PRIVACY LEVEL AGREEMENTS
}

\author{
V. Diamantopoulou, H. Mouratidis
}

\begin{abstract}
With the enforcement of the General Data Protection Regulation, any entity seeking compliance to specific privacy-and security-related requirements, the adoption of Privacy by Design and Security by Design principles can be considered as a legal obligation for any such entity processing EU citizens' personal data. A formal way to support Data Controllers towards their compliance to the new Regulation could be the use of a Privacy Level Agreement (PLA), a mutual agreement of the privacy settings between a Data Controller and a Data Subject, that supports privacy management, by analysing privacy threats, vulnerabilities and Information Systems' trust relationships. However, the concept of PLA has only been proposed on a theoretical level. In this paper, we propose a novel reference architecture to enable PLA management in practice, and we report on the application and evaluation of PLA management. To this aim, two different domains have been selected acting as real-life case studies, the public administration and the healthcare, where special categories of personal data is processed. The results of this evaluation are rather positive, indicating that the adoption of such an agreement promotes the transparency of an organisation while enhances Data Subjects' trust.
\end{abstract}

Keywords: Privacy Level Agreement, Security Requirements Engineering, Privacy Requirements Engineering, Practical Evaluation. 


\section{Introduction}

Globally, Public Authorities and private organisations offer an increasing number of e-services to individuals (i.e. service consumers, citizens, patients). As a result, Information Systems (IS) are developed for different areas of e-services (e.g., healthcare, registration services), they operate in a way that supports governmental and industrial initiatives aiming to provide cheaper, faster and more democratic public services (Homburg, 2008), improving transparency of service consumers' data sharing. However, in the development process of such IS, authorities face important challenges related to the privacy of individuals' data (Loader, 2003). This is becoming even more important with the enforcement of the General Data Protection Regulation (GDPR) that requires compliance to specific privacy- and securityrelated principles. Within the context of this Regulation, organisations that provide e-services or keep personal data, are known as Data Controllers (and Data Processors, but for simplicity reasons throughout the paper we are using the term 'Data Controllers'), while those receiving e-services and having the ownership of the data are the Data Subjects. Data Controllers determine the purposes and the means of processing of the personal data they manage, while Data Processors process personal data on behalf of the Data Controllers (GDPR/Art. 4). Both Data Controllers and Data Processors are accountable for managing the personal data they possess in a way that they respect the privacy of the Data Subjects. The GDPR defines clear obligations and responsibilities for the Data Controllers and Data Processors (GDPR/Art.24, Art.28). In this context, it is important that Data Controllers, through their IS, are able to clearly specify Data Subjects' privacy needs, provide them with feedback on how their data is shared and inform them of potential data privacy conflicts. Moreover, Data Controllers should enable Data Subjects to understand potential threats and vulnerabilities to their privacy requirements, as well as trust relationships that might endanger their privacy. It has been argued in the literature (Diamantopoulou et al., 2017; Diamantopoulou et al., 2017b; Cloud Security Alliance, Privacy Level Agreement Working Group, 2013) that such challenges can be addressed through the use of a Privacy Level Agreement (PLA), which supports a mutual agreement between a Data Controller and a Data Subject, regarding the Data Subject's privacy needs and the transparency of their data sharing.

In the context of cloud services, in order to facilitate the satisfaction of a client's privacy requirements, the notion of PLA has been used (Cloud Security Alliance, Privacy Level Agreement Working Group, 2013; Ahmadian et al., 2015) as part of a Service Level Agreement (SLA) (Bouman et al., 1999; Keller and Ludwig, 2003) as a bilateral agreement between cloud service providers and their clients on how and to what degree the data of the latter should be protected. In the area of IS engineering, we have presented in previous work (omitted reference for the review) a language that supports the modelling of PLAs for Data Controllers' IS services and a theoretical analysis of how the PLA can be used to support implementation of a schema towards the GDPR compliance (omitted reference for the review).

In this paper, we build on our previous work, and we make the following contributions: First, we propose a reference architecture for PLA management, both at design time and during runtime. This is important because it allows the creation of PLAs as digital contracts, between Data Subjects and Data Controllers, which can by analysed at design time and used at runtime to guarantee that the Data Subjects' privacy is respected, based on their privacy preferences. Next, we perform an empirical evaluation across two domains (public administration and health care), which is focused on evaluating the applicability of the PLA and on the identification of potential benefits and difficulties raised from its usage. This is the first effort in the literature to practically evaluate PLAs in the context of Information Systems (IS) services.

The remainder of the paper is set out as follows: Section 2 discuses related work, while Section 3 presents a brief overview of the PLA structure and introduces the reference architecture. Section 4 illustrates the application of our work on case studies from the Public Administration and Healthcare domains, and Section 5 evaluates the results of this. Finally, Section 6 summarises the conclusions and raises issues for further research. 


\section{Related Work}

Conceptually, the proposed architecture consists of two distinct parts, the managerial one and the adaptation of security and privacy requirements engineering methodologies. In order to realise the importance and the novelty of the proposed architecture, the related work consists of two parts; the first is related with the management of the security and privacy requirements of individuals and the second focuses on the methodologies capturing such requirements.

\subsection{Management of security and privacy requirements}

The term of the Privacy Level Agreement is not a new one. The Privacy Level Agreement Working Group of the Cloud Security Alliance has defined a PLA in the context of cloud services (Cloud Security Alliance, Privacy Level Agreement Working Group, 2013). Similarly, the concept of PLA has been presented by DErrico and Pearson (2015) as a standardised way for cloud providers to describe their data protection practices. In the same way to the Cloud Security Alliance proposal, this work focuses on the cloud environment and the PLA is considered as a means for the cloud providers to ensure that their privacy policy is communicated to the service consumers. However, these works are limited only to the examination of privacy aspects of cloud provision and do not provide support for specification of user (e.g., citizen) preferences and needs or ways to define privacy threats and vulnerabilities related to these needs, important aspects of the PLA of this work.

A study of Ahmadian et al., (2015) presents a tool-based approach that facilitates the ISO27001 certification process for cloud service providers, appropriate for SMEs. The authors present the ClouDAT framework, a cloud-specific risk assessment process that allows the automatic generation of ISO27001 compliant documentation, based on the outcomes of the risk assessment. The PLA is used as an input to their tool in order to perform security checks. Compared to our work, this framework focuses on the security analysis on cloud environment, with little emphasis on privacy requirements been given. Also, this approach does not support Data Subjects (e.g., citizens, patients) specifying their privacy preferences and needs.

The idea of a standardised way for web sites to communicate with users about their privacy policies in a standard machine-readable format has been introduced by the Platform of Privacy Preferences (P3P) Project (Platform for Privacy Preferences (P3P) Project, 2016). This standard enables web browsers and other user agents to interpret privacy policies on behalf of their users, assisting them to decide when they exchange data with web sites. However, P3P was designed for static environments where users' privacy preferences are not expected to change, and it also provides limited support for specification of privacy threats and vulnerabilities that might endanger the privacy needs.

A study of Drogkaris et al., (2013) proposes an architecture that promotes the employment of privacy policies and preferences. The authors introduce the Privacy Controller Agent for storing and comparing service providers' privacy policies and user privacy preferences. However, this work does not provide an agreement between two entities (e.g., PA and citizens) but rather an architecture to define privacy policies.

On the other hand, the literature provides many examples of works that focus on the specification of Service Level Agreements (SLAs) which refer to the mutual agreement that defines the obligations and the requirements both of a service provider and a customer (e.g., Bouman et al., 1999; Keller and Ludwig, 2003; García et al., 2017; Mohamed et al., 2017). In contrast to the PLA concept, an SLA does not take into account privacy aspects of the agreement between a service provider and a service consumer.

Consequently, the proposed architecture for the establishment of a PLA, either in an already existing system or in a new one, differs from the solutions analysed above. The proposed architecture takes into consideration the requirements of the natural persons (under the examined scope), providing thus the ability for the development of a personalised agreement between the data controller and the data subject. Moreover, the proposed solution is a dynamic one, allowing updates when necessary, either by the Data Controller or by the Data Subject. Finally, most of the existing solutions do not pay enough focus on the 
privacy requirements, creating thus a gap in the completeness of the provided system from the perspective of the satisfaction of requirements imposed by the GDPR.

We have also contributed to the state-of-the-art by formally specifying the concept of the PLA, based on an XML schema, which enables its automated use (omitted reference for the review). In addition, we have extended our work (omitted reference for the review) so the PLA can be used to support the GDPR, providing the metamodel of the PLA. Finally, an application of the PLA in healthcare domain is presented in (omitted reference for the review), focusing mainly on the functionalities of the PLA management platform.

\subsection{Security and privacy requirements methodologies}

Various approaches have been proposed in the literature for systematically capturing security and privacy requirements. In the area of privacy requirements, the Privacy Safeguard (PriS) (Kalloniatis et al., 2008) methodology enables the elicitation of privacy requirements in the software design phase, where privacy requirements are modelled as organisational goals. Next, in (Spiekermann and Cranor, 2009) the authors adopt the concepts of privacy-by-policy and privacy-by-architecture, and propose a threesphere model of user privacy concerns, relating it to system operations (i.e. data transfer, storage and processing). Additionally, the Modelling and Analysis of Privacy-aware Systems (MAPaS) framework (Colombo and Ferrari, 2012) is a framework for modelling requirements for privacy-aware systems. In (Deng et al., 2011) the authors present LINDDUN, a privacy threat analysis framework which, in its first release, aimed at the elicitation and fulfilment of privacy requirements in software-based systems. The process that LINDDUN follows is that a data flow diagram (DFD) of the system is designed and then the identified privacy threats are related to DFD elements. Privacy threat trees and misuse cases are used for the collection of threat scenarios that might affect the system. Moreover, this methodology supports the elicitation of the final privacy requirements and the selection of appropriate privacy enhancing technologies. The final stage of this methodology is the prioritisation and validation of privacy threats through risk assessment. LINDDUN also provides a map that connects privacy enhancing technologies with each privacy requirement, facilitating thus the system designers to select the most appropriate techniques that are able to satisfy privacy requirements.

Regarding security requirements methodologies, literature provides numerous works that have been developed. Indicatively, we present SQUARE (Security Quality Requirements Engineering) methodology (Mead and Stehney, 2005) which is a risk-driven method that supports the elicitation, categorisation, prioritisation and inspection of the security requirements through a number of specific steps. It also supports the performance of risk assessment to verify the tolerance of a system against possible threats. Nexrt in (Faßbender et al., 2014a,b) the authors propose the Problem-based Security Requirements Elicitation (PresSuRE) Methodology that facilitates the identification of security needs during requirements analysis of software systems, providing a computer security threat recognition and then the development of security requirements. Ahmed and Matulevicius (2014) introduced an asset-based approach for the elicitation of security goals from business process models which are then translated into security requirements. This method follows a sequence of steps. During the first step, an early analysis is performed that identifies the business assets that are valuable and must be protected against security risks. The second step is dedicated to the elicitation of security requirements during the examination of the security risk of business assets. The final stage is the elicitation of security requirements which results to the generation of business rules that satisfy security goals of the system under examination. In (Salini and Kanmani, 2013) the authors propose Model Oriented Security Requirements Engineering (MOSRE) framework for Web Applications which considers security requirements at the early stages of the development process, covering all phases of requirements engineering and suggesting the specification of the security requirements in addition to the specification of systems requirements. Differently than these works, our study provides a start-to-end implementation of a security and privacy management approach that takes into account the $\mathrm{PbD}$ principles, starting with the elicitation of the user privacy needs and ending with the provision of PA online services, and the security by design principles, by conducting 
security analysis of the IS of the service provider, allowing the detection of threats and facilitating the selection of suitable security mechanisms to mitigate potential attacks.

Considering the two areas analysed above, the proposed architecture presents a holistic flow of actions, which incorporates the elicitation of security and privacy requirements engineering methodologies in order to provide a clear view of the examined system, as well as to provide a dynamic interaction allowing the re-identification of the PLA, when necessary.

\section{Reference Architecture for Privacy Level Agreements Man- agement}

In the context of this work, we define a PLA as the mutual agreement of the privacy settings between a Data Controller (i.e. Public Administration (PA)) and a Data Subject (i.e. citizen), where the former will commit to provide and maintain these settings throughout the provision of the service. The PLA, which embodies the privacy policy that must be applied, is delivered in a form of a structured agreement that consists of fields, each of them capturing important and obligatory information with regards to privacy of Data Subjects' data. Moreover, an XML schema has been proposed (omitted reference for the review) to enable the creation and management of machine-readable PLAs, allowing its utilisation by diverse/agnostic IS, thus addressing interoperability issues. The XML schema enables automated processing of the information included in the PLA, for every entity understanding this schema. In this section, we present an overview of the PLA structure, as proposed in (omitted reference for the review) to support understanding of the rest of the paper. We then describe the proposed reference architecture for PLA management, across two levels: Design Time and Runtime. In briefly, at design time we meet the requirements' specifications and the generation of the PLA and at runtime the PLA enforcement is executed. Detailed analysis on this follows is sections 3.2 and 3.3, respectively.

\subsection{Brief Overview of PLA structure}

The structure of the PLA contains two sections, the first with information related to the Data Controller and the second to the Data Subject. In turn, each section contains a number of fields that include information related to the privacy of the Data Subjects' data. Most of the fields are associated with requirements of the GDPR (omitted reference for the review) in order to demonstrate their necessity and their appropriateness. Remaining fields relate to the information flow between the Data Controller and the Data Subject. It is worth mentioning in this point that the responsible role, from the Data Controller's side, should be the Data Protection Officer (DPO). The Data Controller's PLA section can and is proposed to be supported by the appointed DPO. This way, the DPO will have the responsibility related to the completion of all the necessary actions described below, for creating and maintaining the PLA. However, in the architecture presented below, a DPO actor is not explicitly depicted, for generality purposes, as not all the Data Controllers are obliged to appoint a DPO. It can be considered that in the proposed architecture the DPO (or corresponding role) is managing all the described processes.

Data Controller Section The field Identity presents the contact details of the Data Controller and the responsible administrator, i.e. name, place of establishment. The field Data specifies which personal data the Data Subject needs to provide to the Data Controller. Next, the field Data Processing Ways provides information about processing and storing Data Subjects' data. The field Data Sharing Preferences contains information about third parties that can have access to Data Subjects' data. The field Data Privacy Measures specifies the technical, physical, and organisational measures in place to protect Data Subjects' personal data against any destruction or loss, alteration, unauthorised use, modification, disclosure of access, and any other unlawful form of processing. The field Privacy Threat Analysis provides the threat analysis of the Data Controller's privacy requirements. The field Trust Analysis accordingly, provides the trust analysis of the Data Controller's privacy requirements. Last, the field Law Compliance gives information on whether privacy requirements are compliant with corresponding privacy national or EU laws and regulations. 
Data Subject Section The field National Public Authority contains the necessary details of the Authority responsible for protecting Data Subjects' personal data rights. The field Data Subject Privacy Preferences has the privacy preferences of the Data Subject that have been collected by the Data Controller. The field History Based Assessment consists of an analysis of the Data Subjects' privacy preferences and the generation of a prediction of the possible outcomes of subsequent requests. Last, the field Data Value contains the average of i) the Data Subject's perspective concerning their data, ii) the valuation that the Data Controller provides, and iii) the average valuation of all the Data Subjects.

\subsection{Privacy Level Agreement at Design Time}

The IS of a Data Controller which offers e-services has to be set up in a way to facilitate the management of the PLA. To this end, the Data Controller uses appropriate methods and tools that will enable them to capture the relevant information during the design time of the system-to-be. A reference architecture that implements the relevant process during the design time is depicted in Figure 1.

The Data Subject who wishes to use the Data Controller's e-service has to provide only the necessary data for the provision of the e-service. This data is then stored to the Data Controller database. Once the data is captured, the Data Controller needs to specify their processing rights, representing the purpose for which the data is collected (GDPR/Art. 5). So, the Data Controller captures their Privacy Requirements. This can be achieved by any method and tool available in the literature.

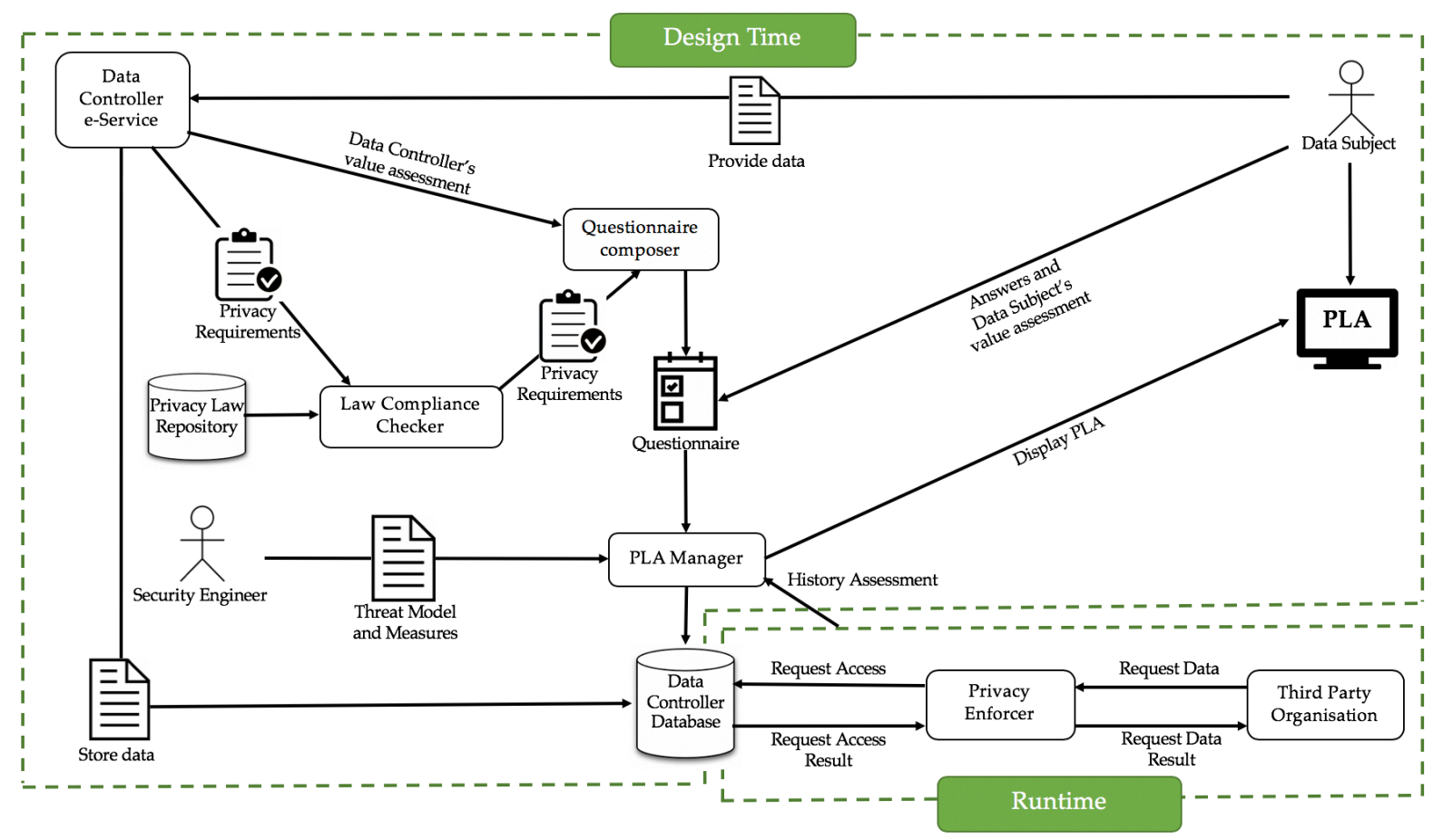

Figure 1. Reference Architecture for PLA Management

Furthermore, the relevant laws and regulations at a national and international level are encoded and stored to the Privacy Law Repository. Compliance of the privacy requirements with such laws and regulations has to be checked and verified by the Law Compliance Checker. The latter collects i) the specified privacy requirements and ii) the relevant laws, in order to shape the final privacy requirements. This part regards the specification of how Data Controllers manage and use the personal data of Data Subjects and the specification of constraints imposed by national, European, or international legislation. Also, at this stage, the Data Controller proceeds to a valuation of the collected Data Subjects' data in order to provide this information later in the PLA.

Next, a Data Controller's Security Engineer conducts a security analysis of the system under examination. The output of this analysis is a threat model that depicts the potential threats that can exploit system vulnerabilities and cause privacy breaches. These threats can be mitigated by identifying additional 
Security and Privacy Measures. Furthermore, the aforementioned analysis can be used for the specification of an appropriate system architecture that has to be developed by the Security Engineer along with an examination that this architecture does not violate the privacy requirements.

Finally, the last activity of the Data Controller during the design time is to define the questions of the questionnaire which will be used by the Questionnaire Composer. These questions are related with the identification of the entities that are allowed to have access to the personal data, with the corresponding services that this data will be associated with, and with the definition of the period that the data will be stored for (when this period is not being determined by the legislation). After the Questionnaire Composer has received this information, the Questionnaire is composed and released. After the questionnaire has been released, the Data Subject is requested to fill it in, stating their privacy preferences and providing their own assessment, revealing their perception about the value of their personal data. The completed questionnaire is then collected by the PLA Manager which generates the PLA, stores it to the Data Controller Database and displays it to the Data Subject. The PLA is provided to the Data Subject either in a pdf form or via a web URL.

\subsection{Privacy Level Agreement at Runtime}

The relevant information during runtime is also depicted in Figure 1. Once the IS of the Data Controller is implemented and put in operation, the Data Subject can register to the system in order to use its eservices. At this point, the PLA between the Data Subject and the Data Controller is generated, since all the relevant information has been captured. To support the PLA generation, we have defined an XML schema ${ }^{1}$ which supports machine-readable analysis (omitted reference for the review).

Access to Data Subject's data will depend on the individual PLA of the Data Subject. Every time a Third-Party Organisation is requesting access to the Data Subject data, the request is received by the Privacy Enforcer. The protection of the privacy of the citizens' data is achieved by respecting privacy policies, using as basis the privacy preferences defined by the citizens. The privacy enforcer parses the policies defined by the Data Subject's answers, and according to them, the requests are either allowed or denied. These requests are then sent to the PLA manager, creating History Assessment information which captures the ratio of allowed/denied requests of Data Subjects' data. This ratio is then depicted in the PLA, keeping Data Subjects updated regarding the level of openness of their data. This information is also useful to the Data Controllers in order to revise their security architecture of their IS.

The PLA is a dynamic document which is continuously updated. These updates can be triggered because of any alteration that the Data Subject wishes to make regarding their privacy preferences, or because of the aggregation of the results of the value assessment other Data Subjects have provided, or because of the privacy enforcer's results regarding the allowed/denied requests of Data Subject's data. Finally, on the Data Controller's side, the PLA can also change because of a change to the architecture of the IS, which might be a result of a business activity (e.g., collaboration with another organisation, delivery of a new or an extended e-service, etc.).

\section{$4 \quad$ Case Study}

The aim of this section is to illustrate the applicability of the PLA and to identify potential benefits and difficulties by its adoption. In order to have objective results, we evaluate the application of the PLA management at real case studies of two different pilots, run in the following two domains: Public Administration and Healthcare domain. The challenge that these domains have to face is that their online administration services lack the trust of end-users (European Commission, 2015; Bouckaert, 2012). This study aims to reveal potential difficulties found in these domains in the adoption and use of e-services. Additionally, with these case studies, this work will demonstrate the advantages of the adoption of the PLA, both on the Data Controllers' and the Data Subjects' side. We analysed two different domains,

\footnotetext{
${ }^{1}$ http://www.sense-brighton.eu/xml_pla/
} 
with different scenarios, trying to achieve a holistic case study (Zainal, 2007), by evaluating the overall proposed architecture and by analysing two applicable scenarios in these two different domains.

\subsection{Public Administration domain}

The first pilot examines the applicability of the PLA in the context of a governmental IS. A local PA, the Municipality of Athens (MoA), provides e-government services, using an IS that stores and manages personal data of Athenian citizens, namely MACS (Municipality of Athens Computer Services). The main purpose of MACS is to interconnect with collaborative to MoA organisations, such as hospitals, banks, sport facilities, and many others, and also to store and transmit information necessary for the completion of a citizen's request (e.g., the issue of a birth certificate) without requiring a citizen's physical presence.

Although MACS supports multiple e-services, for simplicity reasons, we focus on the e-service related to a citizen's subscription to a local fitness centre. MoA offers $15 \%$ discount to Athenian citizens, i.e. the ones who can prove that they permanently live in the city of Athens. As proof of their locality, citizens are required to provide their birth certificate, issued by MoA. An Athenian citizen requests the issue of their birth certificate using the MACS system, which directly transmits the required information to the IS of the fitness centre, as part of the e-government services in MoA. It is also required that the citizen provides a medical certificate regarding their health condition, especially if they suffer from any heart disorders or to prove that they do not have any skin condition. The citizen has to visit their physician in order to be provided with this certificate. If the citizen has recently received a medical certificate, MACS is able to retrieve it from the hospital's database and, with the consent of the citizen, to forward it to the IS of the fitness centre. In this scenario, the MACS will handle the birth- and the medical certificate of a citizen. Based on the one-stop concept (Tambouris and Wimmer, 2008; Sedek et al., 2011), i.e. sharing information across multiple IS belonging to different governmental authorities and organisations, the MACS is interconnected with both other PAs and service providers, so the citizen has to provide their privacy preferences regarding the information that they wish to share and how. Moreover, the administrators of MoA must ensure transparency of the data sharing process with other organisations, thus minimising any reluctance of citizens in sharing their personal data, and eventually in using e-services of MoA through MACS. In order for citizens to take the right decision regarding the sharing of their data, they should be aware of i) the relevant mechanisms MoA administrators apply to their IS in order to protect citizens privacy, and ii) the value of their data.

\subsection{Healthcare domain}

The second pilot, from the healthcare domain, provides different challenges since it involves crossborder exchange of medical data, which is of more sensitive nature, and also there are emergency cases where the Data Subject (i.e. patient) is not able to get involved in the process of the provision of consent. This pilot involves two paediatric clinics, namely Ospedale Pediatrico Bambino Gesù (OPBG) and Hospital Infantil Universitario Niño Jesús (HIUNJ), from two different countries, Italy and Spain, respectively. These clinics use a telemedicine platform to exchange medical information of patients. The processes in telemedicine services fall within the sensitive data being processed by electronic instruments, which are currently regulated by the provisions of Directive 2002/58/EC (European Commission: Directive 2002/58/EC of the European Parliament and of the Council, 2002). The methods and the solutions necessary to ensure confidentiality, integrity and availability of data should therefore be adopted in accordance with the security measures explicitly provided in the Directive 95/46/EC (European Commission: Directive 95/46/EC of the European Parliament and of the Council, 1995), covered under the GDPR (European Parliament: Regulation (EU) 2016/679 of the European Parliament and of the Council of 27 April 2016 on the protection of natural persons with regard to the processing of personal data and on the free movement of such data, and repealing Directive 95/46/EC (General Data Protection Regulation, 2016)) and the new regulation replacing the Directive 2002/58/EC, which can be found in (European Commission: Proposal for a Regulation of the European Parliament and of the Council, 2012). In our scenario, an Italian patient suffers from a disease which is characterised by doctors as quite rare and 
complex enough. The physicians who provide health care to the patient, decide to discuss this case with a specialist group in another hospital, HIUNJ, in Spain. Due to several circumstances, it is impossible for the physicians of the two countries to arrange a meeting with physical presence. Thus, tele-consultation is arranged in order for the physicians to decide the most appropriate diagnostic procedures and therapy. The physicians at OPBG conduct their diagnosis, and they produce a medical report which is accompanied with medical images of the patient. This data is retrieved by the specialised group at HIUNJ via an OPBG web application. After the HIUNJ have received the necessary data, they are able to provide their assessment for the case, confirming whether the first diagnosis is correct or not.

\subsection{Privacy Level Agreement Management in the case of the MoA}

The process of the PLA management that is followed is the same in both case studies. For simplicity reasons, in this work we demonstrate, in the rest of this section, only the application of our work on the PA pilot, although we discuss in Section 5 the evaluation of the work on both cases.

To support the management of PLAs both at design and runtime levels, as discussed in Section 3, Data Controllers need to make use of appropriate tools and techniques to capture the organisational structure of their system and perform privacy, security and trust analysis. Although the selection of such tools and techniques depends on each organisation, the MoA pilot made used of tools developed as part of the VisiOn EU project (VisiOn Privacy Platform, 2016) platform.

Design time. At the design level, three main activities are performed that form input to the PLA, as described in Section 3. In particular, the Questionnaire Creation, the law compliance checks and the privacy and threat analysis. In the MoA pilot, to provide input to the PLA from the privacy and threat analysis, MoA Security Engineers modelled privacy requirements (Gharib et al., 2016) of their MACS system, using the STS modelling language (Salnitri et al., 2015) of the VisiOn platform. Based on that analysis, business processes were designed, using the SecBPMN2 tool (Salnitri et al., 2016), that are implemented by the MACS IS, capturing also any identified interaction with other systems. For example, the activity Allow access was modelled, in order to provide permission for reading the medical certificate and storing it in the local database, allowing citizens to access the fitness centre's IS.

Then, the MoA Security Engineers made use of the SecTro tool, a modelling tool supporting the analysis and consideration of security, from the early stages of the development process (Mouratidis et al., 2016) to enrich the STS-ml model with privacy and security requirements and relevant privacy objectives. As an example, through that analysis, it was identified that the MACS privacy constraint regarding access to medical certificate can be satisfied through the undetectability privacy objective.

Privacy mechanisms stored in a library of the tool which has been enhanced with privacy patterns (Diamantopoulou et al., 2017c) These patterns are used to identify appropriate privacy solutions, such as smart cards and permission management. The results of this analysis were then used as input in the corresponding field of the PLA, indicating the specific privacy and security mechanisms that MoA has to use, assuring the citizen of the level of their privacy protection, thus increasing their trust in MoA eservices.

Next, the MoA Security Engineers constructed a trust model using the JTrust tool (Pavlidis et al., 2014) in order to identify dependencies among the system's stakeholders. In the context of sociotechnical systems, four types have been identified; the first type is the experiential trust and originates from previous experience that one entity gains from the interaction of another entity; the second type is the reported trust, which emerges from a third party that reports that an entity is trustworthy; the third type of trust is the normative trust, which originates from the norms established by the system environment (e.g., a law or a regulation that enforce an entity to fulfil its goal); the fourth type of trust is the external trust, which emerges from external origins, outside the environment of the examined system, such as governments, supervisory and any other authorities. In our case, the citizen depends on the IS of the fitness centre to keep their medical certificate confidential. This dependency implies a trust relationship between the citizen and the IS of the fitness centre and is justified with Reported Trust, i.e. MoA reports that the IS of the fitness centre can be trusted to keep the medical certificate confidential. It is worth noting that in cases where this analysis reports lack of trust, control mechanisms are added. 
After that, Security Engineers added security annotations on the model of the system's architecture. The CARiSMA tool (Jürjens, 2002) is used for the design of the models by using UMLsec, and performs checks to validate that the architecture satisfies the security requirements. In our scenario, the transmission of a document by the MACS to the IS of the fitness centre was annotated with the security requirement of confidentiality. There, a transmission annotation was added to both actors, to verify that the checks performed by the tools are successful. This ensures the implementation of all the necessary security measures that guarantee the protection of citizens' data.

The last part of the analysis that MoA Security Engineers conducted was compliance with the relevant laws and regulations, at national and European level, to prove to citizens that they are compliant to the law. For the MoA pilot, the LIONoso tool (Battiti and Brunato, 2014) was used as the law compliance checker to i) specify how MoA uses/manages citizen's data; ii) specify the constraints imposed by laws and regulations and iii) verify compliance of the data management specified by MoA with the constraints specified in the aforementioned specification of laws and regulations. MoA Security Engineers inserted, in a machine-readable format, the relevant national and EU privacy laws related to the privacy preservation of personal and sensitive data. Then, they specified the operations that are applied over this data, e.g., MoA produces birth certificate. Finally, MoA Security Engineers used the Data Value Tool (DVT) of the VisiOn platform to assess citizen's personal data. This value was then compared with both MoA expectations and citizen's perspective, and the results are visualised to the users through the PLA.

When MoA Security Engineers completed the aforementioned analysis, they proceeded with the creation of an appropriate, privacy related, questionnaires so that the Data Subjects (in our case, citizens) using the MoA services could define their privacy preferences. For the MoA pilot, the Dynamic Audit Engine (DAE) tool of the VisiOn platform played the role of the questionnaire composer, assisting MoA in easily creating questionnaires. Once the citizen registered to the MoA e-service, they had to answer this questionnaire. The answers to those questionnaires formed the last input to the PLA.

Runtime. This phase regards the enforcement of the PLA. The preferences of the citizen, as they have been recorded in the PLA, are parsed by the Privacy Enforcer. In the pilot described in this case, two tools were used, namely the Privacy Agreement Enforcer (PAE) and the Media Aware Network Element (MANE). PAE tool is responsible for creating privacy policies which are formulated by the privacy preferences defined by citizens, after they filled in the questionnaire. Moreover, PAE evaluates requests for accessing private data against these policies, checking the policies that apply to that specific data and enforces the results. MANE is responsible for monitoring and filtering network traffic, acting as a second layer of data protection by applying access rules according to the data received from PAE. In case that we have a request by a third-party organisation to gain access to a citizen's personal data, PAE receives this request and checks if this activity is allowed, according to the generated PLA (which contains citizen's privacy preferences). If the citizen has denied MoA the right to share their personal data e.g., for commercial purposes, MoA applies this policy. The result of PAE is then forwarded to MANE which automatically stops future requests to this specific information. Also, a notification (e.g., via SMS) about the attempts to access their data and the corresponding results is sent to the citizen. In this way, the citizen is continuously updated, and this positively affects their trust in MoA e-services. Finally, the results of the actions of both PAE and MANE are forwarded to LIONoso, which has already been described in the design time. LIONoso updates the number of requests to citizen's data, thus formulating a history-based assessment percentage.

\section{$5 \quad$ Evaluation}

This section aims to evaluate the usefulness of the PLA and to provide insights about its applicability. This evaluation has been conducted under two perspectives. On the one hand, at a technical level, we assess PLA's usability and potential challenges that the end users came across during its management. On the other hand, from a social point of view, we evaluate the PLA management reference architecture regarding the impact, for both Data Controllers and Data Subjects, on the protection of their personal data and the level of trust of Data Subjects to Data Controllers during the provision of their data. 


\subsection{Evaluation Method}

PLA management is achieved through the use of a series of tools, each one responsible for a specific part of it. More specifically, the tools that are necessary and contribute to the management of the PLA, during the design time i) conduct privacy analysis on Data Controllers' IS, by analysing threats, vulnerabilities, and trust relationships of their IS with other ones, ii) create the questionnaire that allows Data Subjects to provide/declare their privacy preferences, iii) allow the assessment of the value of Data Subjects' data, and, during runtime, they v) enforce and monitor Data Subjects' privacy preferences. The management of the PLA is accompanied with a novel, quite technologically challenging solution. Such an approach can be either accepted or rejected by the end-users, according to their behaviour towards the solution, after testing its use. For this reason, we decided to support the evaluation process based on the Technology Acceptance Model (TAM) (Davis, 1989; Venkatesh and Davis, 2000). TAM approach is an information systems theory that models how users come to accept and use a technology. The model suggests that when users are presented with a new technology, a number of factors influence their decision on how and when they will use it. Notably, the approach introduces two determinants: i) Perceived Usefulness (PU), which is defined as "the degree to which a person believes that using a particular system would enhance their job performance", and ii) Perceived Ease-Of-Use (PEOU), which is defined as "the degree to which a person believes that using a particular system would be free from effort". Therefore, PU provides insight on how useful the technological approach behind PLA management is, while PEOU on how easy it is to use. This evaluation method aims to include parameters that will quantify end-users' perspective, on what extent the release of such technology is necessary and could cover their need. Furthermore, it will provide insights regarding the required effort that the endusers have to employ to use the technological solution that supports the management of the PLA, since users tend to adopt technologies that not only are effective but can also be apprehended in an effortless way.

\section{$5.2 \quad$ Results}

For the two pilots, the means of the evaluation was an online survey, which was designed under the TAM approach, and took place between February and June 2017. The questions aim to mainly confirm the increased perception of trust in online public services, both for the Data Controllers and for Data Subjects. Thus, the end-users report the level of privacy on their personal data, so they validate their willingness to share data while feeling secure and protected from the adoption of the PLA in the corresponding e-service.

The first part of the questionnaire is common for both Data Controllers and Data Subjects, including additionally three demographic questions on sex, age, and education level. Next, there are questions solely for Data Controllers and solely for Data Subjects. The Public Administration questionnaire included 18 questions for PAs and 20 for citizens, while the Healthcare questionnaire included 13 questions for the administrators and 18 for patients. The questionnaires were distinct for each of the two pilots as the case studies were different as well, i.e. the case study for the public administration domain describes an interaction taking place between a citizen and a public body, when the first wishes to use an online service offered by the later, while the case study for the healthcare domain describes a crossborder exchange of medical data in an emergency situation. In addition, the nature of the exchanged data in these case studies is of different value (the scenario of the public administration domain focuses on personal data while the scenario of the healthcare domain focuses on special categories of personal data, i.e. health data, that requires different management). However, we present below, in Table 1 and Table 2, respectively, the questions that triggered significant statistical responses and results, and may offer valuable findings for this study. All questions are given in multiple choice format, with answers possible in the following 5-point Likert scale; totally disagree, disagree, neither agree/nor disagree, agree, totally agree. For simplicity reasons, we provide only the top three percentages per question.

\begin{tabular}{|l|l|l|}
\hline Criterion & Question & The Respondents: \\
\hline
\end{tabular}




\begin{tabular}{|c|c|c|}
\hline PEU, Supportability & $\begin{array}{l}\text { Do you find that the process of the PLA } \\
\text { management is designed for all levels of } \\
\text { users, irrespective of their technical back- } \\
\text { ground? }\end{array}$ & $\begin{array}{l}\text { Agree } 47,22 \% \text {, Neither agree/nor } \\
\text { disagree } 30,56 \% \text {, Disagree } 18,06 \%\end{array}$ \\
\hline PU, Reliability, Security & $\begin{array}{l}\text { Is PLA management platform useful for } \\
\text { providing innovative services to Data } \\
\text { Subjects, as far as privacy is concerned? }\end{array}$ & $\begin{array}{l}\text { Agree } 62,50 \%, \text { Totally agree } \\
22,22 \%, \text { Neither agree/nor disagree } \\
15,28 \%\end{array}$ \\
\hline $\begin{array}{l}\text { PU, Reliability, Threat, } \\
\text { Information Control }\end{array}$ & $\begin{array}{l}\text { Would PLA management platform im- } \\
\text { prove privacy in public services provided } \\
\text { to Data Subjects? }\end{array}$ & $\begin{array}{l}\text { Agree } 58,33 \% \text {, Totally agree } \\
23,61 \% \text {, Neither agree/nor disagree } \\
15,28 \%\end{array}$ \\
\hline PU, Reliability & $\begin{array}{l}\text { Would PLA increase trust to public ser- } \\
\text { vices? }\end{array}$ & $\begin{array}{l}\text { Agree } 51,39 \% \text {, Totally agree } \\
25,00 \% \\
\text { Neither agree/nor disagree } 22,22 \%\end{array}$ \\
\hline $\begin{array}{l}\text { PEOU, Perceived Ease of } \\
\text { Learning }\end{array}$ & $\begin{array}{l}\text { Are you confident that you can complete a } \\
\text { process for the management of a PLA? }\end{array}$ & $\begin{array}{l}\text { Agree } 62,50 \%, \text { Neither agree/nor } \\
\text { disagree } 22,22 \% \text {, Totally agree } \\
09,72 \%\end{array}$ \\
\hline \multicolumn{3}{|c|}{ Solely Administrators Questions } \\
\hline $\begin{array}{l}\text { PEOU, Supportability, } \\
\text { Performance }\end{array}$ & $\begin{array}{l}\text { Is PLA management platform easy to inte- } \\
\text { grate with existing public services? }\end{array}$ & $\begin{array}{l}\text { Agree } 43,24 \% \text {, Neither agree/nor } \\
\text { disagree } 43,24 \% \text {, Disagree } 10,81 \%\end{array}$ \\
\hline Platform requirements & $\begin{array}{l}\text { Does PLA management platform com- } \\
\text { municate securely with other public bod- } \\
\text { ies to execute the request? }\end{array}$ & $\begin{array}{l}\text { Agree } 54,05 \% \text {, Neither agree/nor } \\
\text { disagree } 29,73 \% \text {, Totally agree } \\
13,51 \%\end{array}$ \\
\hline \multicolumn{3}{|c|}{ Solely Citizens Questions } \\
\hline PU, Information Control & $\begin{array}{l}\text { Would PLA make it easy to understand } \\
\text { the actual values of our personal data? }\end{array}$ & $\begin{array}{l}\text { Agree } 51,43 \% \text {, Neither agree/nor } \\
\text { disagree } 20,00 \% \text {, Disagree } 17,14 \%\end{array}$ \\
\hline Platform Requirements & $\begin{array}{l}\text { Does PLA assist Data Subjects regarding } \\
\text { the preferred level of privacy? }\end{array}$ & $\begin{array}{l}\text { Agree } 71,43 \%, \text { Neither agree/nor } \\
\text { disagree } 20,00 \% \text {, Totally agree } \\
08,57 \%\end{array}$ \\
\hline PU & Would you use PLA for public services? & $\begin{array}{l}\text { Agree } 71,43 \% \text {, Neither agree/nor } \\
\text { disagree } 17,14 \% \text {, Disagree } 08,57 \%\end{array}$ \\
\hline
\end{tabular}

Table 1. Questionnaire in Public Administration domain

\begin{tabular}{|l|l|l|}
\hline Criterion & Question & The Respondents: \\
\hline $\begin{array}{l}\text { PEOU, Perceived Ease of } \\
\text { Learning }\end{array}$ & $\begin{array}{l}\text { Is the PLA management platform easy to } \\
\text { use? }\end{array}$ & $\begin{array}{l}\text { Agree } 49,50 \% \text {, Neither agree/nor } \\
\text { disagree 25,80\%, Totally agree } \\
23,70 \%\end{array}$ \\
\hline $\begin{array}{l}\text { PU, Reliability, Threat, In- } \\
\text { formation Control }\end{array}$ & $\begin{array}{l}\text { Before the simulation, did you know the } \\
\text { privacy and security aspects of health } \\
\text { data? }\end{array}$ & $\begin{array}{l}\text { Neutral 35,30\%, Not really 34,20\%, } \\
\text { Somewhat } 15,80 \%\end{array}$ \\
\hline $\begin{array}{l}\text { PU, Information Control, } \\
\text { Reliability }\end{array}$ & $\begin{array}{l}\text { Do you think this process has raised your } \\
\text { awareness of the privacy issues by } \\
\text { providing you with greater awareness } \\
\text { and understanding of the importance of } \\
\text { protecting your data? }\end{array}$ & $\begin{array}{l}\text { Agree } 46,30 \%, \text { Neither agree/nor } \\
\text { disagree } 18,90 \% \text {, Totally agree } \\
17,40 \%\end{array}$ \\
\hline PU, Supportability & $\begin{array}{l}\text { Do you think that PLA management plat- } \\
\text { form can guarantee greater privacy and } \\
\text { security while exchanging health data? }\end{array}$ & $\begin{array}{l}\text { Agree } 44,20 \%, \text { Totally agree } \\
30,50 \%, \text { Neither agree/nor disagree } \\
15,80 \%\end{array}$ \\
\hline \multicolumn{2}{|c|}{ Solely Patients Questions } \\
\hline PU, Information Control & $\begin{array}{l}\text { Do you think PLA management platform } \\
\text { allows patients greater control over }\end{array}$ & $\begin{array}{l}\text { Agree } 49,50 \%, \text { Totally agree } \\
23,70 \%, \text { Neither agree/nor disagree } \\
14,70 \%\end{array}$ \\
\hline
\end{tabular}




\begin{tabular}{|l|l|l|}
\hline & $\begin{array}{l}\text { privacy using constraints on health data } \\
\text { transmission? }\end{array}$ & \\
\hline $\begin{array}{l}\text { PU, Information Control, } \\
\text { Risks }\end{array}$ & $\begin{array}{l}\text { Do you think this process has made you } \\
\text { aware of the potential risks or benefits of } \\
\text { consciously compiling the consent for } \\
\text { the transmission of health data? }\end{array}$ & $\begin{array}{l}\text { Agree } 56,30 \% \text {, Totally agree } \\
24,20 \%, \text { Neither agree/nor disagree } \\
12,60 \%\end{array}$ \\
\hline $\begin{array}{l}\text { PEOU, Perceived Ease of } \\
\text { Learning, Supportability }\end{array}$ & $\begin{array}{l}\text { Do you think this process can be useful } \\
\text { for patients with particular clinical needs } \\
\text { or problems, such as reduced mobility? }\end{array}$ & $\begin{array}{l}\text { Agree } 42,10 \% \text {, Totally agree } \\
31,10 \%, 40 \%\end{array}$ \\
& $\begin{array}{l}\text { Do you think the PLA offers a complete } \\
\text { insight on privacy and security issues? }\end{array}$ & $\begin{array}{l}\text { Agree } 46,80 \% \text {, Totally agree } \\
36,30 \%, \text { Disagree } 8,40 \%\end{array}$ \\
\hline PU, Information Control & Dogree \\
\hline
\end{tabular}

Table 2. Questionnaire in Healthcare domain

The evaluation of the process regarding the generation of the PLA in the context of the presented case study followed a systematic approach, combining on one hand the objectives the users set, i.e. the improvement of users' privacy, the level of security, and their trust when they use PAs services, and on the other hand, the requirements related to the development of the PLA, using a series of tools. Each requirement could be either technical, and therefore its fulfilment could be verified by checking the existence of the functionality(ies) implemented, or could refer to a human perspective, such as usability of the corresponding tools. The users evaluated the whole process of the identification of security, privacy, and trust requirements as easy, even though they were not familiar with such tools and methodologies at the start of the project. They mentioned some difficulties during their first interaction with the tools, the effort they put in order to integrate the VisiOn platform in their already existing platforms and systems, and in understanding the functionalities of each tool. However, they realised the importance of such a platform, that could support the services they deliver, as they can demonstrate they take into consideration citizens' privacy, by securing their sociotechnical systems, respecting the privacy requirements, and by analysing the trust relationships they have developed with external entities. Finally, a remarkable comment was that the users considered the proposed work as a method that could act as an additional trust layer between the existing services that the MoA provides to the citizens of Athens, acting as a facilitator between a public body and a citizen, increasing trust to offered public services.

The following two paragraphs provide a thorough analysis of the results of the case studies for the public administration domain and for the healthcare one.

Public Administration domain In this pilot, the sources for engaging participants - that had already been contacted and recruited during pilot preparation - derived from both private companies as well as in the public sector among the Municipality of Athens, other municipalities and bodies of local administration. The goal was to engage mainly participants that are occupied as Public Administrators but have as well a strong background in Information Technology e.g., Security Engineers, professionals in the IT Department of municipalities, System Administrators, etc. The distribution of participants was in majority males, however the difference with female participants is not so significant (31 out of 72 were female and 41 male). The most populated age-group was between 30-40 years (almost 57\%) and 20\% were from 40-50 years. Most participants have the age profile of an active working professional, which is also verified by the level of education, where the majority of participants, $19,44 \%$ and $63 \%$, have undergraduate or postgraduate studies, respectively.

From a technical perspective, the questions that capture the ease of use of the PLA management platform and the process of the management of a PLA outcome interesting results. The majority of the participants agrees that they can easily complete the process of a PLA management. In addition, this process is designed for all the various users, irrespective of their technical background. These two findings indicate that the PLA management platform is well-designed and does not require possession of specialised technical skills. Of course, there is always room for improvement. PLA management platform is a novel technical approach and requires continuous improvement to be approved by its end-users. Approaches facilitating the usability (Pattakou et al., 2018) of such framework should be always taken into 
consideration at the early stages of their design. The question related to its integration and secure communication with existing systems highlights the interoperability issues that every new system faces during their integration with legacy systems. These difficulties have to be taken into account and individually examined for each system. The appropriate parameterisation of the IS should be taken into account, as well as the demanded effort and time for the successful completion of this process.

Focusing on the usefulness and the impact that the adoption of the PLA has, the findings are rather positive. The PLA management platform is considered quite useful for providing innovative services to individuals, with respect to privacy. Moreover, the adoption of the PLA contributes to the improvement of privacy issues related to public services. Next, the percentage of respondents $(51,39 \%)$ who indicate the increase of trust to public services provides us the confidence that the adoption of the PLA is actually beneficial for Public Administrators, who increase their trustworthiness, attracting thus more citizens to use their e-services. The last years, public sector has put a lot of effort to promote transparency (Obama, B., 2011), making their processes understandable, accurate, and reusable. PLA can contribute towards the improvement of public sector trustworthiness, and consequently, towards the increase of citizens' trust. Finally, PLA offers the possibility of scalable provision of consent, allowing citizens to define their privacy preferences, which can be updated, if necessary. This attribute has been assessed positively by individuals, who, hereafter have realised the importance of their personal data.

Healthcare domain This pilot includes 190 participants from two hospitals, 89 from OPBJ and 101 from HIUNJ. Nearly half of the participants have a job as office worker while nearly $25 \%$ of users are freelancers. More specifically, 22,6\% are freelances, $45,8 \%$ are office workers, $4,7 \%$ have retired, $10,5 \%$ are students, and $4,2 \%$ are unemployed. Cumulatively, there was a balance in the distribution of the respondents, since $48,40 \%$ were male and $51,60 \%$ female. $43,20 \%$ of the participants are between 30 39 years old, percentage that again indicates their active working profile. However, there are significant percentages in higher ages (14,70\% among 40-49 and 50-59, cumulatively), since the patients in these clinics are minors and are represented by their legal guardians. The participants' educational level is mainly on bachelor's degree $(57,90 \%)$ and upper secondary $(36,30 \%)$.

Evaluating the management of the PLA from a technical point of view, we can see that the majority of the respondents find this process easy. Of course, this percentage should not be examined solely, but in combination with the high educational level of the majority of participants. The privacy analysis of an organisation's IS is a demanding process that requires a substantial background of knowledge. However, our participants were not familiar with security and privacy engineering methodologies. This parameter indicates that the management platform is user-friendly and users who are not familiar with such tools and methodologies are able to use it successfully, delivering insight on privacy and security issues for their organisation.

Regarding the usefulness of the PLA and the impact that it has on the individuals, the results are more than positive. Despite that participants were not aware of the privacy and security aspects of health data (the majority of them (almost 70\%)), they mentioned that through their involvement of this process, they were able to deeply understand the importance of protecting the data they provide in such an organisation. Moreover, they feel confident that the hospital can guarantee greater privacy and security while exchanging health data. We highlight here that the healthcare domain pilot contains many peculiarities, such as the provision of not only personal but also sensitive data, the emergency cases that often demand quick responds and decisions, the necessity of cross-border exchange of patients' data, and the representation of the patients by their legal guardians, which also reflects the principle of GDPR related to the parental consent. Thus, it is quite meaningful that with the use of PLA, the patients have realised the criticality behind the provision of their personal and sensitive data or their biometrics. Another important benefit from the adoption of the PLA is that it is useful for patients with particular clinical needs or difficulties. One of the major benefits of an online healthcare service is to simplify some operations for patients living in remote areas or who face particular clinical difficulties that could benefit the most from the use of a web platform, and the PLA can contribute towards this.

Natural persons, and specifically after the enforcement of the GDPR, have the right to be informed about the processing of the data they provide to public bodies. The inclusion, in the evaluation process, of a 
Public Administration body shed light to the attention that must be given to interoperability issues that arise, when a new system needs to communicate with already existing ones. On the other hand, the inclusion of hospitals in the evaluation of the PLA revealed that special attention must be provided by the Data Controllers when they process special categories of personal data. Besides, the necessity for fortifying the communication channel when the data is cross-border transmitted, is imperative. This is an obligation that must be fulfilled by both organisations involved in this transmission. Results of this study also indicate that both these domains need the use of the proposed architecture as in both cases the clients/recipients of their services are positively inclined in use of such service that would increase the trustworthiness levels of a service.

\section{Conclusions}

This paper has presented a novel reference architecture for the management of Privacy Level Agreements and a practical evaluation of this work. The evaluation has been conducted on the public administration domain and on the healthcare domain, where personal and special categories of personal data, i.e. sensitive ones, is being handled. Moreover, another peculiarity that these domains have is that individuals (i.e. citizens, patients) do not have other option than providing their personal data in order to deliver the corresponding e-services. However, it is fundamental right of the Data Subjects, when they provide their data to any organisation, to know who processes their data, if this data is being shared to other entities, and how long the organisation retains the data, if this goes beyond the obligatory by the legislation retention period.

Additionally, the Data Controllers and the Data Processors are obliged to be compliant with the GDPR, when they process personal data. The proposed work, that provides as outcome the generation of the PLA, can be considered as a privacy-by-design supporting architecture, capturing the privacy preferences of the users and enforcing them to the corresponding personal data processing systems.

The results of the evaluation indicate that appropriate management of PLAs allows Data Controllers (public bodies, private organisations) to demonstrate that they have taken all the necessary actions to mitigate the identified threats, and also to demonstrate transparency with regards to processing and sharing of Data Subjects' data. With respect to Article 32 of the GDPR, Data Controllers, with the use of the proposed tools described in the design time of this process, are able to demonstrate that they have implemented appropriate technical and organisational measures for ensuring a defined level of security. On the other hand, PLA is a means of enhancing trust of Data Subjects to Data Controllers, thus decreasing the number of users that are reluctant to use governmental and other public e-services. The information that the PLA includes can contribute to the satisfaction of the right to information of the Data Subjects (GDPR/Articles 13-14), and to the limitation of the requested data (data minimisation).

The results of this work are useful for practitioners and researchers. At theoretical level, the process described in this work can be further extended to capture more concepts related with the satisfaction of the GDPR requirements, such as the satisfaction of additional rights of the Data Subjects, i.e. right of access, right to rectification, restriction of processing, right to object. From a practical perspective, future work can focus on the development of tools and methods to deal with aspects of the GDPR that PLAs are not supporting, such as privacy risks assessment, privacy complaints, and breach notifications.

The presented work is part of a larger effort to develop a framework to support compliance with the GDPR. The ultimate goal is to finally make transparency and accountability inherent characteristics of all activities related to citizens' data within Data Controllers. Monitoring how this data is used after it has been provided to the Data Controllers is also one of the main functionalities of this work. This, along with the enforcement of PLA, plays a critical role in the maximisation of transparency and accountability, that is important specifically for the public administration for ensuring that services offered in this domain will gain users' trust. 


\section{References}

Ahmadian, A. S., Coerschulte, F., and Jürjens, J. (2015). Supporting the Security Certification and Privacy Level Agreements in the Context of Clouds. In International Symposium on Business Modeling and Software Design, Springer, Cham, 80-95.

Ahmed, N., and Matulevicius, R. (2014). A Method for Eliciting Security Requirements from the Business Process Models. In CAiSE (Forum/Doctoral Consortium) (pp. 57-64).

Battiti, R., and Brunato, M. (2014). The LION way. Machine Learning plus Intelligent Optimization. LIONlab, University of Trento, Italy, 94.

Bouckaert, G. (2012). Trust and public administration. Administration, 60(1), 91-115.

Bouman, J., Trienekens, J., and Van der Zwan, M. (1999). Specification of service level agreements, clarifying concepts on the basis of practical research. In Software Technology and Engineering Practice, 1999. STEP'99. Proceedings (pp. 169-178). IEEE.

Colombo, P. and Ferrari, E. (2012). Towards a modeling and analysis framework for privacy-aware systems. In Privacy, Security, Risk and Trust (PASSAT), 2012 International Conference on Privacy, Security, Risk and Trust and 2012 International Conference on Social Computing (SocialCom). IEEE, 81-90.

CSA (2013): Privacy level agreement outline for the sale of cloud services in the European Union. Tech. rep., Cloud Security Alliance, Privacy Level Agreement Working Group

Davis, F. D. (1989). Perceived usefulness, perceived ease of use, and user acceptance of information technology. MIS quarterly, 319-340.

Deng, M., Wuyts, K., Scandariato, R., Preneel, B., and Joosen, W. (2011). A privacy threat analysis framework: supporting the elicitation and fulfillment of privacy requirements. Requirements Engineering, 16(1), 3-32.

DErrico, M., and Pearson, S. (2015). Towards a formalised representation for the technical enforcement of privacy level agreements. In Cloud Engineering (IC2E), 2015 IEEE International Conference, IEEE, $422-427$.

Omitted omitted reference for the review

Diamantopoulou, V., Angelopoulos, K., Pavlidis, M., and Mouratidis, H. (2017). A Metamodel for GDPR-based Privacy Level Agreements. In ER Forum/Demos (pp. 285-291).

Diamantopoulou, V., Kalloniatis, C., Gritzalis, S., and Mouratidis, H. (2017b). Supporting privacy by design using privacy process patterns. In IFIP International Conference on ICT Systems Security and Privacy Protection (pp. 491-505). Springer, LNCS.

Diamantopoulou, V., Pavlidis, M., and Mouratidis, H. (2017c). Privacy level agreements for public administration information systems. In Proceedings of the CAiSE Forum 2017 29th International Conference on Advanced Information Systems Engineering, X. Franh, J. Ralyté, R. Matulevičius, C. Salinesi, and R. Wieringa, (eds), Essen, Germany, CEUR LNCS, 97-104.

Drogkaris, P., Gritzalis, S., and Lambrinoudakis, C. (2013). Employing privacy policies and preferences in modern e-government environments. International Journal of Electronic Governance, 6(2), 101116.

European Commission: Directive 2002/58/EC of the European Parliament and of the Council (July 2002), URL: http://ec.europa.eu/justice/data-protection/law/files/recast_20091219_en.pdf (visited on 23/05/2018).

European Commission: Directive 95/46/EC of the European Parliament and of the Council, URL: http://eur-lex.europa.eu/legal-content/EN/TXT/?uri=CELEX (visited on 23/01/2019).

European Commission: Eurobarometer 431 - Data Protection Report. Technical report (2015), URL: http://ec.europa.eu/commfrontoffice/publicopinion/archives/ebs/ebs_431_sum_en.pdf (visited on 25/04/2019)

European Commission: Proposal for a regulation of the European Parliament and of the Council (January 2012), URL: http://eur-lex.europa.eu/legal-content/en/ALL/?uri=CELEX:52012PC0011 (visited on 23/01/2019).

European Parliament: Regulation (EU) 2016/679 of the European Parliament and of the Council of 27 April 2016 on the protection of natural persons with regard to the processing of personal data and on 
the free movement of such data, and repealing Directive 95/46/EU (General Data Protection Regulation) (2016), URL: http://eur-lex.europa.eu/legal-content/EN/TXT/PDF/?uri=CELEX:32016R0679\&from=en31995L0046 (visited on 23/05/2018).

Faßbender, S., Heisel, M., and Meis, R. (2014a). Functional requirements under security pressure. In 9th International Conference on Software Paradigm Trends (ICSOFT-PT) IEEE, 5-16.

Faßbender, S., Heisel, M., and Meis, R. (2014b). Problem-based security requirements elicitation and refinement with pressure. In International Conference on Software Technologies. Springer, Cham, 311-330

García, J. M., Fernandez, P., Pedrinaci, C., Resinas, M., Cardoso, J., and Ruiz-Cortés, A. (2017). Modeling service level agreements with linked USDL agreement. IEEE Transactions on Services Computing, 10(1), 52-65.

Gharib, M., Salnitri, M., Paja, E., Giorgini, P., Mouratidis, H., Pavlidis, M., Ruiz, J. F., Fernandez, S., Della Siria, A. (2016). Privacy requirements: findings and lessons learned in developing a privacy platform. In 2016 IEEE 24th International Requirements Engineering Conference (RE) (pp. 256265). IEEE.

Homburg, V. (2008). Understanding e-government: Information systems in public administration. Routledge.

Jürjens, J. (2002). UMLsec: Extending UML for secure systems development. In International Conference on The Unified Modeling Language. Springer, Berlin, Heidelberg, 412-425.

Kalloniatis, C., Kavakli, E. and Gritzalis. S. (2008). Addressing privacy requirements in system design: the PriS method. Requirements Engineering 13, 3, 241-255.

Keller, A., and Ludwig, H. (2003). The WSLA framework: Specifying and monitoring service level agreements for web services. Journal of Network and Systems Management, 11(1), 57-81.

Loader, B. D. (2003). The governance of cyberspace. In The governance of cyberspace (pp. 13-30). Routledge.

Mead, N. R., and Stehney, T. (2005). Security quality requirements engineering (SQUARE) methodology (Vol. 30, No. 4, ACM, 1-7.

Mohamed, M., Anya, O., Tata, S., Mandagere, N., Baracaldo, N., and Ludwig, H. (2017). rSLA: An Approach for Managing Service Level Agreements in Cloud Environments. International Journal of Cooperative Information Systems, 26(02), 1742003.

Mouratidis, H., Argyropoulos, N., and Shei, S. (2016). Security requirements engineering for cloud computing: The secure tropos approach. In Domain-specific conceptual modelling. Springer, Cham. 357-380.

Obama, B. (2011). Transparency and open government. Presidential Memorandum (January 21, 2012), http://www. whitehouse. gov/thepress-office/transparency-and-open-government.

Pattakou, A., Mavroeidi, A. G., Diamantopoulou, V., Kalloniatis, C., \& Gritzalis, S. (2018). Towards the Design of Usable Privacy by Design Methodologies. In 2018 IEEE 5th International Workshop on Evolving Security \& Privacy Requirements Engineering (ESPRE) (pp. 1-8). IEEE.

Pavlidis, M., Islam, S., Mouratidis, H., and Kearney, P. (2014). Modeling trust relationships for developing trustworthy information systems. International Journal of Information System Modeling and Design (IJISMD), 5(1), 25-48.

Salini, P., and Kanmani, S. (2013). Model oriented security requirements engineering (MOSRE) framework for web applications. In Advances in Computing and Information Technology, Springer, Berlin, Heidelberg, 341-353.

Salnitri, M., Paja, E., and Giorgini, P. (2016). Maintaining secure business processes in light of sociotechnical systems' evolution. In Requirements Engineering Conference Workshops (REW), IEEE International, IEEE, 155-164

Salnitri, M., Paja, E., Poggianella, M., and Giorgini, P. (2015). STS-Tool 3.0: Maintaining Security in Socio-Technical Systems. In CAiSE Forum, 205-212.

Sedek, K. A., Sulaiman, S., and Omar, M. A. (2011). A systematic literature review of interoperable architecture for e-government portals. In Software Engineering (MySEC), 2011 5th Malaysian Conference, IEEE, 82-87. 
Spiekermann, S. and Cranor, L. F. (2009). Engineering privacy. IEEE Transactions on software engineering $35,1,67-82$.

Tambouris, E., and Wimmer, M. (2008). Online one-stop government: a single point of access to public services. In Electronic Government: Concepts, Methodologies, Tools, and Applications, IGI Global, 2805-2829

Venkatesh, V., and Davis, F. D. (2000). A theoretical extension of the technology acceptance model: Four longitudinal field studies. Management science, 46(2), 186-204.

VisiOn: Vision privacy platform (2016), URL: http://www.visioneuproject.eu/ (visited on 23/05/2018).

W.W.W.C.: Platform for Privacy Preferences (P3P) Project (2016), URL: https://www.w3.org/P3P/ (visited on 23/05/2018).

Zainal, Z. (2007). Case study as a research method. Jurnal Kemanusiaan, 5(1). 